Case Report

\title{
A Case of Multiple Myeloma Coexisting with Primary Hyperparathyroidism and Review of the Literature
}

\author{
Nasir Hussain, Moona Khan, Aparna Natarajan, Mubeenkhan Mohammedabdul, \\ Usman Mustafa, Kalpana Yedulla, and Aibek E. Mirrakhimov
}

Saint Joseph Hospital, Resurrection Health Care, Department of Internal Medicine, 2900 North Lake Shore Drive, Chicago, IL 60657, USA

Correspondence should be addressed to Nasir Hussain; connect2nasir@gmail.com

Received 5 January 2013; Accepted 30 January 2013

Academic Editors: G. Di Vagno, C. Gennatas, J. Kleeff, and F. A. Mauri

Copyright ( $\odot 2013$ Nasir Hussain et al. This is an open access article distributed under the Creative Commons Attribution License, which permits unrestricted use, distribution, and reproduction in any medium, provided the original work is properly cited.

Hypercalcemia is a common medical problem with an estimated prevalence of $15 \%$ among hospitalized patients. Multiple myeloma (MM) and primary hyperparathyroidism (PHPT) are among the most common causes of hypercalcemia but coexistence of both pathologic processes in a patient is an extremely rare phenomenon. In this paper we have discussed a patient presenting with this rare phenomenon. We have also provided a comprehensive review of the scientific literature published on codiagnosis of MM and PHPT.

\section{Introduction}

Hypercalcemia is a common clinical problem with an estimated prevalence of $15 \%$ among hospitalized patients [31]. The etiology of hypercalcemia is complex with many factors playing a pathogenic role. From a clinical standpoint, it may present with changes in mental status, generalized weakness, polyuria, and constipation. Multiple myeloma (MM) and primary hyperparathyroidism (PHPT) are among the most common causes of hypercalcemia but coexistence of the two pathologic processes in one patient is an extremely rare phenomenon. In this paper, we have discussed a patient presenting with this rare phenomenon and have reviewed the relevant scientific literature.

\section{Case Presentation}

A 92-year-old Caucasian female with a past medical history of Alzheimer's dementia, seizure disorder, osteoporosis, and osteoarthritis was admitted to the hospital for an evaluation of a new onset confusion and constipation. Review of symptoms during admission was significant for anorexia, weight loss, constipation for the last three weeks, and history of a fall one month prior to the presentation. Family history was significant for $\mathrm{MM}$ in a sister. At the time of presentation, the patient was using donepezil, memantine, vitamin $\mathrm{D}$ with calcium, calcium carbonate (calcium containing antacid), and levetiracetam. Vitals at the time of admission were blood pressure $140 / 58$, pulse 68 , respiratory rate 18 , oxygen saturation $98 \%$ on room air, and temperature 97.4. On physical examination, the patient was alert and oriented in place and person but not in time. Other significant findings were diastolic murmur in right second intercostal space, petechiae over lower extremities, and back tenderness, which the patient attributed to a recent fall. Lumbar spine Xray was done three weeks prior to the presentation that showed degenerative changes with no evidence of fracture. Basic blood workup including complete blood count and comprehensive metabolic panel was done, which revealed anemia, leucopenia, and hypercalcemia. Home medications were held for concerns of hypercalcemia and confusion.

Endocrinology and neurology services were consulted. MRI of the brain was done, which showed lytic lesions as shown in Figure 1. MM was suspected; serum protein electrophoresis (SPEP), urine protein electrophoresis (UPAP) and bone marrow biopsy were done which confirmed the diagnosis of MM (IgG kappa) (International Staging System stage II). Bone marrow biopsy showed mildly hypercellular 


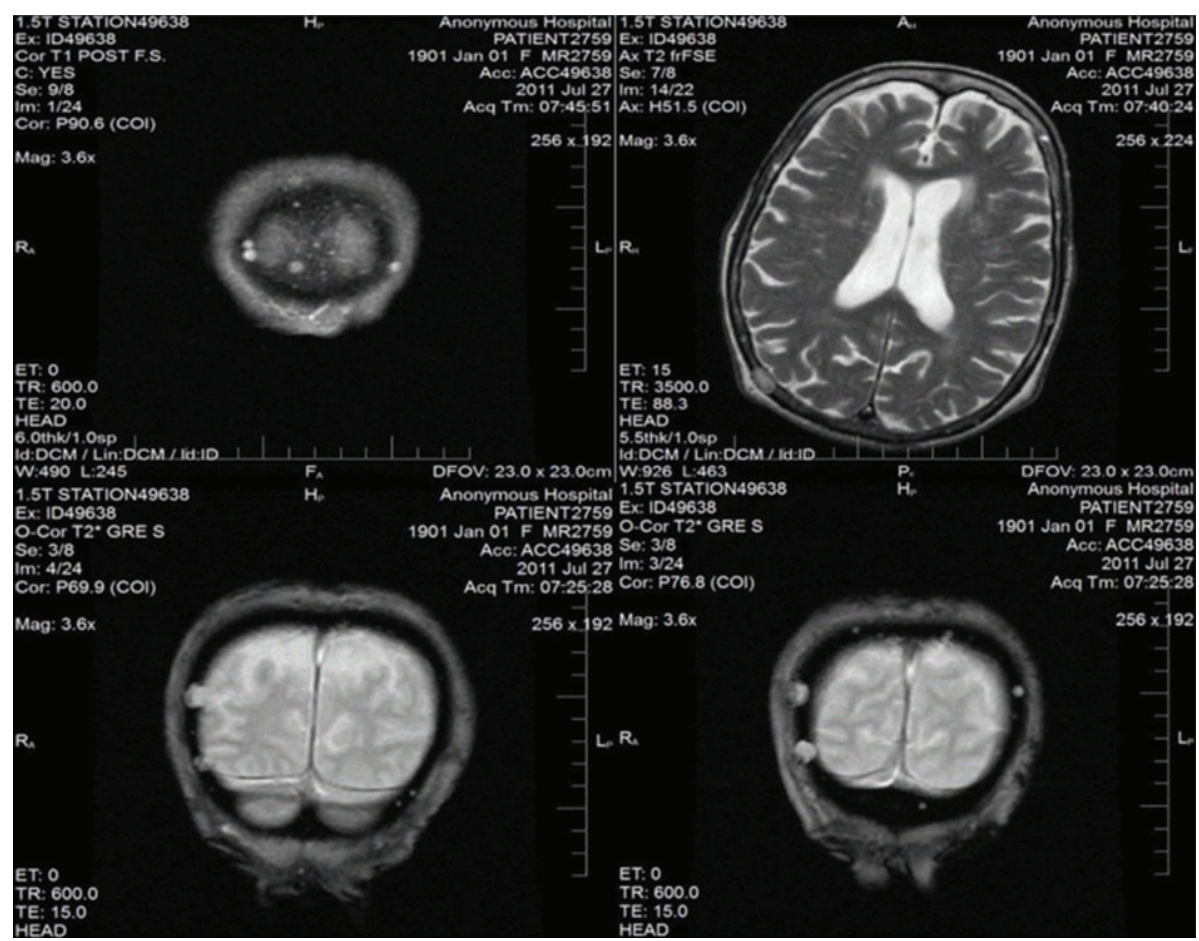

FIGURE 1: MRI demonstrating lytic lesions.

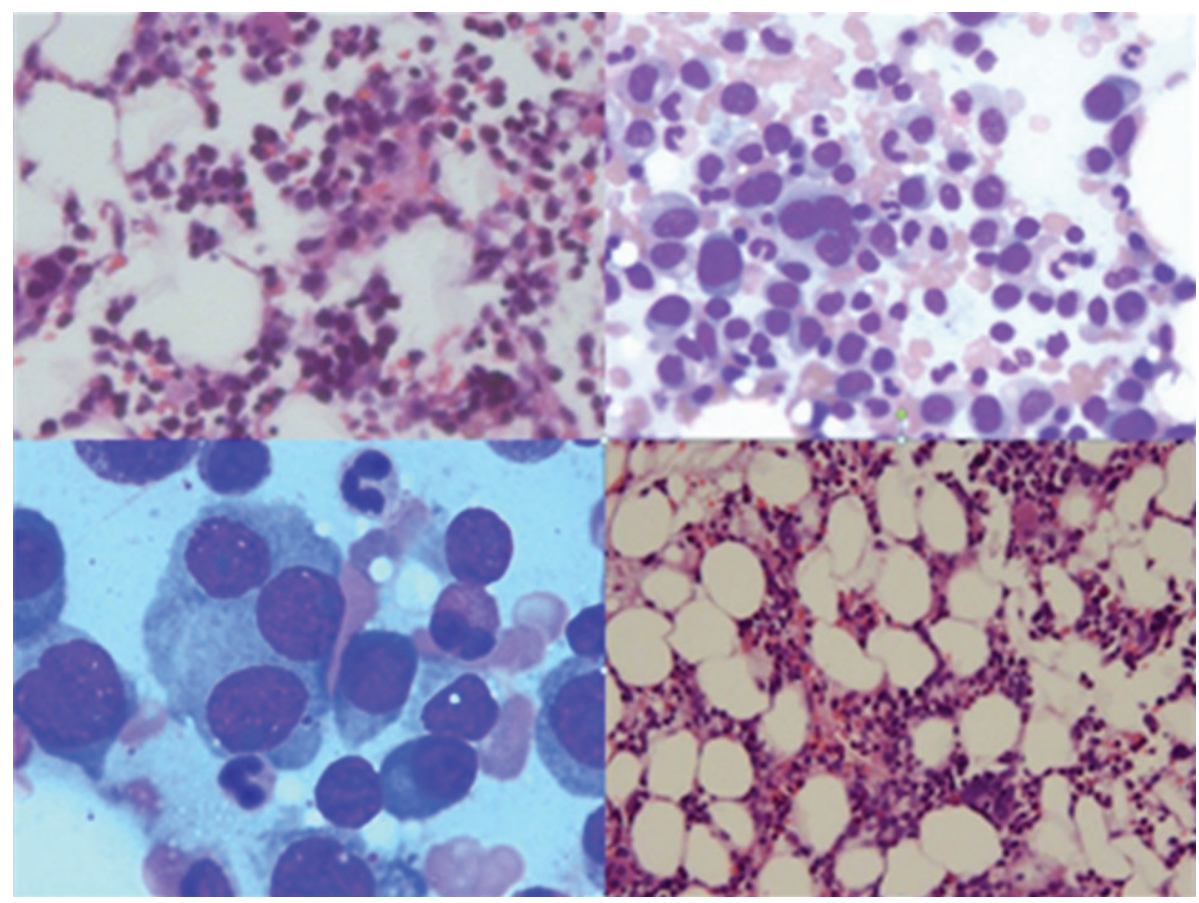

FIGURE 2: Mildly hypercellular bone marrow with plasmacytosis (30\%), consistent with multiple myeloma.

bone marrow with plasmacytosis (30\%) as shown in Figure 2. Skeletal survey showed diffuse lytic lesions throughout long bones, pelvis, and skull (Figure 3). Surprisingly, intact PTH came back high suggesting primary hyperparathyroidism (PHPT). The data on laboratory tests are presented in Table 1.
Hypercalcemia was managed with intravenous hydration, calcitonin, bisphosphonates, and furosemide. The patient was started on melphalan and prednisone, which were later switched to lenalidomide with a high dose of dexamethasone due to a poor treatment response. After one and a half year, 


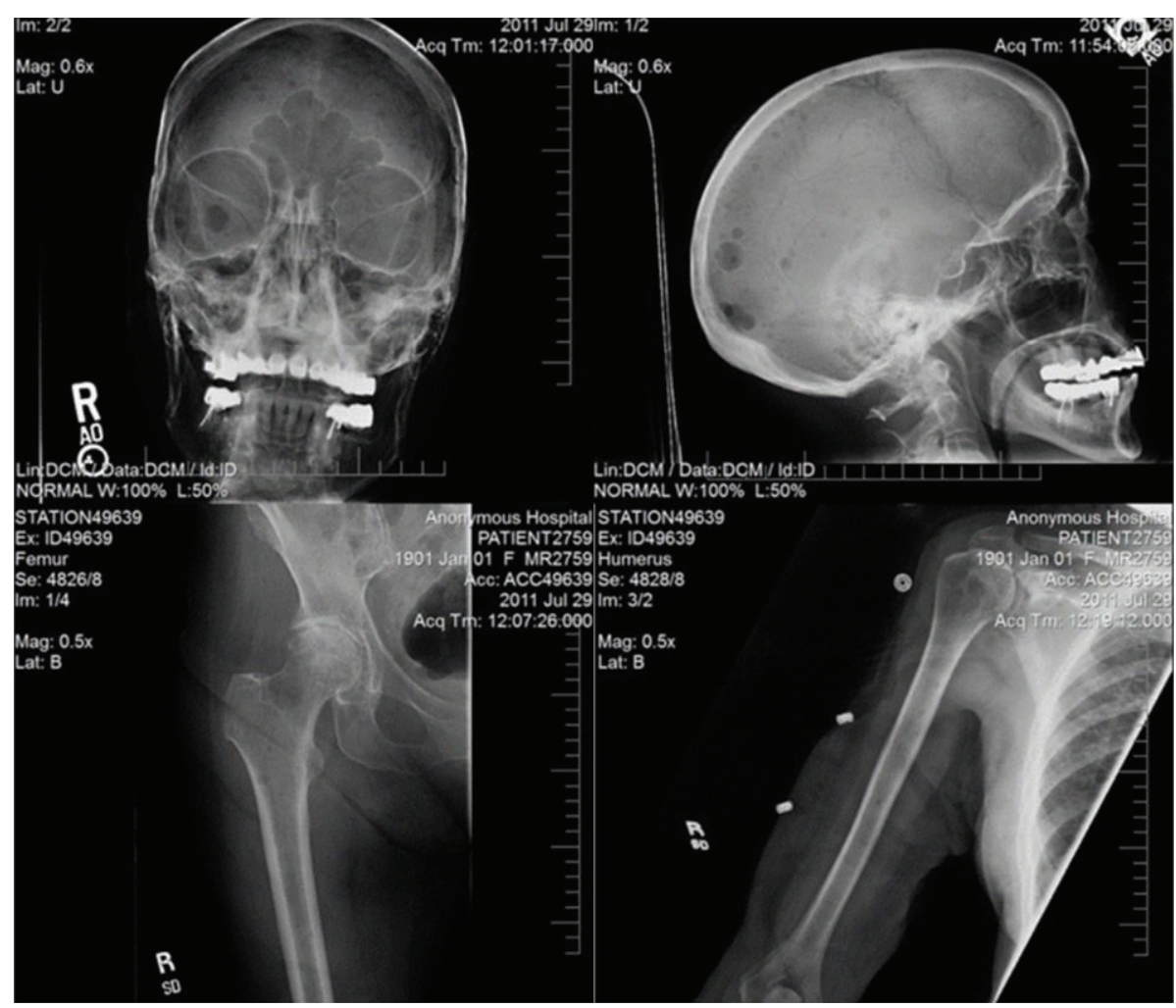

FIGURE 3: Skeletal survey showing lytic lesions in long bones and skulls.

the patient is still following in our outpatient oncology center being on a low dose of lenalidomide with a stable M protein.

\section{Discussion}

Hypercalcemia is common in patients with MM and occurs in $28 \%$ of myeloma cases [32]. MM may cause hypercalcemia through multiple mechanisms. First, plasma cells produce various cytokines, including TNF- $\beta$ and IL-6, that activate osteoclasts and lead to calcium washout from bones to the bloodstream [33]. Second, some studies suggest that MM cells may secrete parathyroid hormone-related peptide similarly to other malignancies, such as squamous cell lung carcinoma $[34,35]$. Third, serum calcium may be falsely elevated because of a binding to immunoglobulin $[36,37]$.

Clubb et al. [38] described first-case linking PHPT and paraproteinemia in 1964. Drezner and Lebovitz were the first who described a case of concomitant MM and PHPT in 1979 [30]. Some researchers speculate that the association between MM and PHTP may not be coincidental [39, 40], although mechanisms explaining codiagnosis are not known. Arnulf et al. showed that the prevalence of monoclonal gammopathy is higher in patients with PHTP as compared to general population [40]. Pest et al. hypothesized that elevated PTH may mediate the induction of MM through the downstream biological effects of IL-6 [1]. This hypothesis was supported by the study performed by Pirih et al., who showed that PTH decreases apoptotic cell death of the hematopoietic stem cells via the IL-6 [41].
PHPT leads to hypercalcemia via direct bone resorption [42] mediated by osteoclasts. Another important mechanism is through an increased calcium absorption in the duodenum and greater reabsorption in the kidneys.

The above-mentioned pathogenic mechanism gives an insight to how PHPT and MM may be linked. Some studies have suggested that calcium may act as a mitogenic factor [43], whereas others suggest that myelomatous proteins may interfere with polypeptide hormone synthesis bind their circulating fractions, and/or block their peripheral effects that may secondarily stimulate parathyroid gland [29]. However, both of these diseases are common among elderly and may share similar risk factors, such as ionizing radiation [44, 45], and a simple coincidence may be the case.

Summary of published cases [1-28] is presented in Table 2. Codiagnosis of PHPT and MM should be suspected in cases of difficult-to-control hypercalcemia. Most of the cases of coexistent MM and PHPT have been observed in females (23 out of 29 reported cases). The youngest patient with codiagnosis was a 45-year-old female and the oldest patient was a 92-year-old female. PHPT is more common in females, whereas the opposite is true for MM. Differences in incidence of the two diseases may explain female preponderance (MM less frequent than PHPT). Initial diagnosis was highly variable, eleven cases had primary diagnosis of hyperparathyroidism, ten had primary diagnosis of MM and seven had both diagnosis made at presentation. The type of immunoglobulin chains of MM observed in all the cases was variable as six patients had light chain $\mathrm{MM}$, remaining 
TABle 1

\begin{tabular}{|c|c|c|}
\hline Result name & Results & $\begin{array}{c}\text { Reference } \\
\text { range }\end{array}$ \\
\hline WBC & $2.9 \mathrm{~K} / \mathrm{mm} \mathrm{cu}$ & $4.2-11.0$ \\
\hline Platelet & $156 \mathrm{~K} / \mathrm{mm} \mathrm{cu}$ & $140-400$ \\
\hline Hemoglobin $\mathrm{Hb}$ & $9.0 \mathrm{~g} / \mathrm{dL}$ & $12.0-15.0$ \\
\hline Hematocrit & $27.1 \%$ & $36.0-47.0$ \\
\hline Reticulocyte & $0.7 \%$ & $0.5-2.8$ \\
\hline Blood urea nitrogen & $22 \mathrm{mg} / \mathrm{dL}$ & $5-20$ \\
\hline Creatinine & $1.11 \mathrm{mg} / \mathrm{dL}$ & $0.0-1.00$ \\
\hline Sodium & $143 \mathrm{mmol} / \mathrm{L}$ & $135-145$ \\
\hline Potassium & $4.0 \mathrm{mmol} / \mathrm{L}$ & $3.4-5.1$ \\
\hline Chloride & $104 \mathrm{mmol} / \mathrm{L}$ & $98-109$ \\
\hline Bicarbonate & $33 \mathrm{mmol} / \mathrm{L}$ & $23-31$ \\
\hline Calcium & $13.3 \mathrm{mg} / \mathrm{dL}$ & $8.4-10.5$ \\
\hline Total protein & $7.0 \mathrm{~g} / \mathrm{dL}$ & $6.4-8.3$ \\
\hline Albumin & $4.0 \mathrm{~g} / \mathrm{dL}$ & $3.4-5.2$ \\
\hline Aspartate amino transferase & $20 \mathrm{IU} / \mathrm{L}$ & $0-32$ \\
\hline Alanine amino transferase & $10 \mathrm{IU} / \mathrm{L}$ & $0-40$ \\
\hline Alkaline phosphatase & $67 \mathrm{IU} / \mathrm{L}$ & $35-104$ \\
\hline Bilirubin total & $0.2 \mathrm{mg} / \mathrm{dL}$ & $0-10.0$ \\
\hline Haptoglobin & $157 \mathrm{mg} / \mathrm{dL}$ & $36-195$ \\
\hline Vitamin B12 & $532 \mathrm{pg} / \mathrm{mL}$ & $211-946$ \\
\hline TSH & $1.160 \mathrm{uIU} / \mathrm{mL}$ & $0.400-5.400$ \\
\hline Vitamin D25 OH & $47.0 \mathrm{ng} / \mathrm{mL}$ & $30.0-100.0$ \\
\hline 25 Hydroxy D3 & $26 \mathrm{pg} / \mathrm{mL}$ & \\
\hline 25 hydroxy D2 & $<8$ & \\
\hline Vitamin D 1,25(OH)2 & 26 & $18-72$ \\
\hline Folate & $>20.0 \mathrm{ng} / \mathrm{mL}$ & $3.1-17.5$ \\
\hline Ferritin & $64 \mathrm{ng} / \mathrm{mL}$ & $13-150$ \\
\hline Phosphorous & $2.8 \mathrm{mg} / \mathrm{dL}$ & $2.0-4.0$ \\
\hline Lactate dehydrogenase & $137 \mathrm{IU} / \mathrm{L}$ & $135-214$ \\
\hline Total iron & $30 \mathrm{ug} / \mathrm{dL}$ & $30-160$ \\
\hline Unsaturated IBC & $234.0 \mathrm{ug} / \mathrm{dL}$ & $110.0-370.0$ \\
\hline Total IBC & 264.0 & $228.0-428.0$ \\
\hline Percentage of iron saturation & $11 \%$ & $20-55$ \\
\hline $\begin{array}{l}\text { PTH intact on day of } \\
\text { presentation }\end{array}$ & $70.5 \mathrm{pg} / \mathrm{mL}$ & $15.0-65.0$ \\
\hline PTH 7 months later & $540.0 \mathrm{pg} / \mathrm{mL}$ & \\
\hline PTH-related protein & $18 \mathrm{pg} / \mathrm{mL}$ & $14-27$ \\
\hline Beta-2 microglobulin & $\begin{array}{c}3.3 \mathrm{mg} / \mathrm{L} \\
(5.8 \mathrm{mg} / \mathrm{L} \text { four } \\
\text { months later })\end{array}$ & $0.8-2.2$ \\
\hline Serum viscosity & $\begin{array}{l}1.5 \text { relative to } \\
\qquad \mathrm{H}_{2} \mathrm{O}\end{array}$ & $1.5-1.9$ \\
\hline PT/INR & $10.6 / 1.0 \mathrm{sec}$ & $9.211 .8 / 0.9-1.1$ \\
\hline APTT & $29 \mathrm{sec}$ & $24-33$ \\
\hline \multicolumn{3}{|l|}{ Immunoglobulins } \\
\hline IGA & $29 \mathrm{mg} / \mathrm{dL}$ & $50-400$ \\
\hline IGG & $\begin{array}{c}692 \mathrm{mg} / \mathrm{dL} \\
(1200,5 \mathrm{months} \\
\text { later })\end{array}$ & $600-1500$ \\
\hline IGM & $6 \mathrm{mg} / \mathrm{dL}$ & $50-300$ \\
\hline
\end{tabular}

TABLE 1: Continued.

\begin{tabular}{lcc}
\hline Result name & Results & $\begin{array}{c}\text { Reference } \\
\text { range }\end{array}$ \\
\hline Free kappa light chains & 1510 & $3.3-19.4 \mathrm{mg} / \mathrm{L}$ \\
Free lambda light chains & 2.4 & $5.7-26.3$ \\
Free Kappa/lambda & 629.17 & $0.26-1.65$
\end{tabular}

Urine protein electrophoresis

Urine volume 24 hours

$1150 \mathrm{~mL} / 24$ hour

Urine-protein electrophoresis (UPE)

$253 \mathrm{mg} / 24$ hour $\quad 0-165$

$\begin{array}{ll}\text { Albumin UPE } & 30.6 \% \\ \text { Alpha1 } & 16.1 \% \\ \text { Alpha2 } & 14.1 \% \\ \text { Beta } & 17.1 \% \\ \text { Gamma } & 22.1 \%\end{array}$

Immunofixation

Free kappa light chains

24-hour-urine protein

$310.5 \mathrm{mg} / 24$
hour

$0-150$

24-hours-urine creatinine

$0.7 \mathrm{~g} / 24$ hour

$0.74-1.57$

24-hour-urine volume

$1150 \mathrm{cc}$

24-hour-urine creatinine

$0.5 \mathrm{~g} / 24$ hour

$0.74-1.57$

24-hour-urine volume

$900 \mathrm{~mL}$ (repeat test)

24-hour-urine calcium

$239 \mathrm{mg} / 24$ hour

$100-300$

Serum protein electrophoresis

Albumin
Alpha 1
Alpha2
Beta
Gamma
Albumin/globulin
M spike
Total protein
Immunofixation
CD56 NK cells
CD 138 marker
Lambda B-cell marker
Kappa B-cell marker
CD45 LCA
CD38 Marker

$3.3 \mathrm{~g} / \mathrm{dL}$

3.1-5.0

$0.3 \mathrm{~g} / \mathrm{dL}$

$0.2-0.5$

$0.7 \mathrm{~g} / \mathrm{dL}$

$0.5-1.1$

$0.6 \mathrm{~g} / \mathrm{dL}$

$0.6-1.1$

$1.5 \mathrm{~g} / \mathrm{dL}$

$0.7-1.7$

1.0

$1.09 \mathrm{~g} / \mathrm{dL}$

$6.5 \mathrm{~g} / \mathrm{dL}$

$6.4-8.3$

Monoclonal paraprotein of class IgG kappa

$\begin{array}{cc}63 \% & 3-35 \\ 26 \% & \\ 1 \% & 1-7 \\ 73 \% & 2-14 \% \\ 98 \% & 92-100 \\ 26 \% & 1-17\end{array}$

Pathology. Normal female bone marrow karyotype. No clonal, structural, or numerical chromosome abnormalities identified. FISH analysis indicates normal hybridization signals with MM probe panel. This excludes majority of chromosome rearrangements known to be associated with MM.

Surgical Pathology. Mildly hyper cellular bone marrow with plasmacytosis consistent with MM.

Leukemia/lymphoma panel. Bone marrow aspirate shows 30-40\% plasma cells with kappa light chain restrictions. (plasma cell dyscrasia).

Peripheral Smear. Lymphocytes with foamy cytoplasm, no rouleaux formation, adequate polys with occasional platelet clumps. 
TABLE 2

\begin{tabular}{|c|c|c|c|c|c|c|c|c|}
\hline$n$ & Author & $\begin{array}{c}\text { Age/ } \\
\text { Gender }\end{array}$ & $\begin{array}{l}\text { Type of } \\
\text { MM }\end{array}$ & $\begin{array}{c}\mathrm{Ca} \\
(\mathrm{mg} / \mathrm{dL})\end{array}$ & Therapy for MM and PHPT & $\begin{array}{l}\text { Parathyroid } \\
\text { histology }\end{array}$ & Outcome & $\begin{array}{c}\text { Initial } \\
\text { diagnosis }\end{array}$ \\
\hline 1 & $\begin{array}{l}\text { Pest et al. } \\
\quad[1]\end{array}$ & $76 \mathrm{~F}$ & $\operatorname{IgA}-?$ & 13.2 & $\begin{array}{l}\text { Hydration, bisphosphonates, Lasix, } \\
\text { melphalan, cyclophosphamide, and } \\
\text { steroids }\end{array}$ & Adenoma & Survived & PHPT \\
\hline 2 & $\begin{array}{l}\text { Rao et al. } \\
\text { [2] }\end{array}$ & $54 \mathrm{M}$ & $\begin{array}{l}\text { IgG- } \\
\text { lambda }\end{array}$ & 11.2 & $\begin{array}{l}\text { Adriamycin, melphalan, prednisone, } \\
\text { cyclophosphamide, and } \\
\text { parathyroidectomy }\end{array}$ & Adenoma & $\begin{array}{l}\text { Died after } 12 \\
\text { years }\end{array}$ & Both \\
\hline 3 & $\begin{array}{l}\text { Jackson and } \\
\text { Orland [3] }\end{array}$ & $45 \mathrm{~F}$ & $\begin{array}{l}\text { IgG- } \\
\text { lambda }\end{array}$ & 17.1 & $\begin{array}{l}\text { Hydration, Lasix, prednisone, and } \\
\text { melphalan }\end{array}$ & Adenoma & - & MM \\
\hline 4 & $\begin{array}{l}\text { Chisholm et } \\
\text { al. [4] }\end{array}$ & $80 \mathrm{M}$ & Kappa & 13.1 & $\begin{array}{l}\text { Parathyroidectomy, radiotherapy, } \\
\text { melphalan, prednisone, vincristine, } \\
\text { carmustine, cyclophosphamide, } \\
\text { hydration, and Lasix }\end{array}$ & $\begin{array}{l}\text { Adenoma } \\
\text { (c-cells) }\end{array}$ & $\begin{array}{l}\text { Died } 2 \text { years } \\
\text { later }\end{array}$ & PHPT \\
\hline 5 & $\begin{array}{c}\text { Francis et al. } \\
{[5]}\end{array}$ & $70 \mathrm{~F}$ & Lambda & 11.6 & $\begin{array}{l}\text { Norethisterone, vincristine, melphalan, } \\
\text { and prednisone }\end{array}$ & Adenoma & $\begin{array}{c}\text { Died } 3 \text { weeks } \\
\text { later }\end{array}$ & PHPT \\
\hline 6 & $\begin{array}{l}\text { Mundis and } \\
\text { kyle [6] }\end{array}$ & $76 \mathrm{~F}$ & IgG-kappa & 11.0 & $\begin{array}{l}\text { Melphalan, prednisone, and } \\
\text { parathyroidectomy }\end{array}$ & $\begin{array}{c}\text { Adenoma } \\
\text { (c-cells) }\end{array}$ & survived & MM \\
\hline 7 & $\begin{array}{l}\text { Stone et al. } \\
{[7]}\end{array}$ & $47 \mathrm{~F}$ & IgA-kappa & 13.7 & $\begin{array}{l}\text { Melphalan, prednisone, radiotherapy, } \\
\text { parathyroidectomy, hydration, and } \\
\text { mithramycin }\end{array}$ & Adenoma & Died & MM \\
\hline 8 & $\begin{array}{l}\text { Hoelzer and } \\
\text { Silverberg } \\
\text { [8] }\end{array}$ & $51 \mathrm{~F}$ & $\begin{array}{l}\text { IgA- } \\
\text { lambda }\end{array}$ & 11.9 & Parathyroidectomy? & $\begin{array}{l}\text { Adenoma } \\
\text { (c-cells) }\end{array}$ & - & PHPT \\
\hline 9 & $\begin{array}{c}\text { Schneider } \\
\text { and Thomas } \\
\text { [9] }\end{array}$ & $74 \mathrm{~F}$ & IgG-kappa & 12.0 & $\begin{array}{l}\text { Melphalan, prednisone, and } \\
\text { parathyroidectomy }\end{array}$ & Adenoma & Survived & MM \\
\hline 10 & $\begin{array}{l}\text { Toussirot et } \\
\text { al. [10] }\end{array}$ & $82 \mathrm{M}$ & Kappa & 15.2 & $\begin{array}{l}\text { Melphalan, prednisone, and } \\
\text { parathyroidectomy }\end{array}$ & Hyperplasia & Died & PHPT \\
\hline 11 & $\begin{array}{l}\text { Goto et al. } \\
\quad[11]\end{array}$ & $73 \mathrm{~F}$ & Kappa & 13.2 & Parathyroidectomy, melphalan & Adenoma & $\begin{array}{l}\text { Died } 1 \text { year } \\
\text { later }\end{array}$ & PHPT \\
\hline 12 & $\begin{array}{l}\text { Otsuka et } \\
\text { al. [12] }\end{array}$ & $77 \mathrm{~F}$ & $\begin{array}{l}\text { IgG- } \\
\text { lambda }\end{array}$ & & $\begin{array}{l}\text { Melphalan, prednisone, bisphosphonates, } \\
\text { calcitonin, and parathyroidectomy }\end{array}$ & $\begin{array}{c}\text { c-cells } \\
\text { hyperplasia }\end{array}$ & Survived & - \\
\hline 13 & $\begin{array}{l}\text { Fery-Blanco } \\
\text { et al. [13] }\end{array}$ & $68 \mathrm{~F}$ & IgG-kappa & 11.28 & ? chemotherapy and surgery refused & Adenoma & Died & Both \\
\hline 14 & $\begin{array}{l}\text { Sarfati et al. } \\
\quad[14]\end{array}$ & $62 \mathrm{~F}$ & IgA-kappa & 16.4 & $\begin{array}{l}\text { Mithramycin, lasix, plasmaphoresis, } \\
\text { Adriamycin, vincristine, prednisone, and } \\
\text { parathyroidectomy }\end{array}$ & Adenoma & Survived & MM \\
\hline 15 & $\begin{array}{l}\text { Rosen et al. } \\
\quad[15]\end{array}$ & $81 \mathrm{M}$ & IgG-kappa & 13.4 & $\begin{array}{l}\text { Hydration, bisphosphonates, melphalan, } \\
\text { prednisone, radiotherapy, needle } \\
\text { aspiration of parathyroid gland, and } \\
\text { refused surgery }\end{array}$ & Adenoma & Survived & MM \\
\hline 16 & $\begin{array}{c}\text { Tomon et al. } \\
{[16]}\end{array}$ & $60 \mathrm{~F}$ & IGA-kappa & - & - & - & - & MM \\
\hline 17 & $\begin{array}{c}\text { Fanari et al. } \\
\text { [17] }\end{array}$ & $59 \mathrm{~F}$ & lambda & 12.7 & $\begin{array}{l}\text { Hydration, bisphosphonates, cinacalcet, } \\
\text { bortezomib and dexamethasone }\end{array}$ & $\begin{array}{l}\text { Possible } \\
\text { Adenoma }\end{array}$ & $\begin{array}{c}\text { Died } 4 \\
\text { months later }\end{array}$ & Both \\
\hline 18 & $\begin{array}{c}\text { Bogas et al. } \\
\text { [18] }\end{array}$ & $72 \mathrm{~F}$ & IgG-kappa & 13.66 & Melphalan, prednisone, and Interferon? & Adenoma & $\begin{array}{l}\text { Died } 4 \text { years } \\
\text { later }\end{array}$ & Both \\
\hline 19 & $\begin{array}{l}\text { Katayama et } \\
\text { al. [19] }\end{array}$ & $50 \mathrm{~F}$ & & & & & & PHPT \\
\hline 20 & $\begin{array}{l}\text { Romagnoli } \\
\text { et al. [20] }\end{array}$ & $70 \mathrm{~F}$ & - & - & $\begin{array}{l}\text { Parathyroidectomy, steroids and } \\
\text { chemotherapy }\end{array}$ & Adenoma & - & $\begin{array}{c}\text { PHPT } \\
(\mathrm{MEN}-1)\end{array}$ \\
\hline 21 & $\begin{array}{l}\text { Toh and } \\
\text { Winocour } \\
\text { et al. [21] }\end{array}$ & $71 \mathrm{M}$ & & 12.0 & $\begin{array}{l}\text { Melphalan, prednisone, and } \\
\text { bisphosphonates }\end{array}$ & & $\begin{array}{l}\text { Died } 6 \text { weeks } \\
\text { later }\end{array}$ & MM \\
\hline 22 & $\begin{array}{l}\text { Sopeña et } \\
\text { al. [22] }\end{array}$ & $77 \mathrm{~F}$ & Kappa (ns) & 12.9 & $\begin{array}{l}\text { Bisphosphonates, refused surgery, or } \\
\text { chemotherapy }\end{array}$ & & $\begin{array}{l}\text { Died } 1 \text { year } \\
\text { later }\end{array}$ & Both \\
\hline
\end{tabular}


TABLe 2: Continued.

\begin{tabular}{|c|c|c|c|c|c|c|c|c|}
\hline$n$ & Author & $\begin{array}{c}\text { Age/ } \\
\text { Gender }\end{array}$ & $\begin{array}{l}\text { Type of } \\
\text { MM }\end{array}$ & $\begin{array}{c}\mathrm{Ca} \\
(\mathrm{mg} / \mathrm{dL})\end{array}$ & Therapy for MM and PHPT & $\begin{array}{c}\text { Parathyroid } \\
\text { histology }\end{array}$ & Outcome & $\begin{array}{c}\text { Initial } \\
\text { diagnosis }\end{array}$ \\
\hline 23 & $\begin{array}{c}\text { Khandwala } \\
\text { and Boctor } \\
{[23]}\end{array}$ & $72 \mathrm{~F}$ & - & $\begin{array}{l}11.7 / \\
16.6^{*}\end{array}$ & $\begin{array}{l}\text { Parathyroidectomy, bisphosphonates, } \\
\text { calcitonin, melphalan, and prednisone }\end{array}$ & Adenoma & - & PHPT \\
\hline 24 & $\begin{array}{l}\text { Patel et al. } \\
\quad[24]\end{array}$ & $73 \mathrm{~F}$ & $\begin{array}{l}\text { IgG- } \\
\text { kappa }\end{array}$ & 13.5 & $\begin{array}{l}\text { Bisphosphonates, steroids, thalidomide, } \\
\text { plicamycin, and parathyroidectomy }\end{array}$ & Adenoma & - & MM \\
\hline 25 & $\begin{array}{l}\text { Avcioglu et } \\
\text { al. [25] }\end{array}$ & $52 \mathrm{~F}$ & IgG-kappa & 12.6 & Parathyroidectomy and steroids & Adenoma & - & Both \\
\hline 26 & $\begin{array}{l}\text { Chowdhury } \\
\text { and } \\
\text { Scarsbrook } \\
\text { et al. [26] }\end{array}$ & $87 \mathrm{~F}$ & - & - & - & - & - & PHPT \\
\hline 27 & $\begin{array}{l}\text { Dalgleish } \\
\text { and } \\
\text { Gatenby } \\
\text { [27] }\end{array}$ & $59 \mathrm{~F}$ & $\begin{array}{l}\text { IgG- } \\
\text { lambda }\end{array}$ & 11.68 & $\begin{array}{l}\text { Hydration, lasix, prednisone, } \\
\text { mithramycin, cyclophosphamide, and } \\
\text { parathyroidectomy }\end{array}$ & Adenoma & Survived & MM \\
\hline 28 & $\begin{array}{l}\text { Peters et al. } \\
\quad[28]\end{array}$ & $73 \mathrm{M}$ & $\begin{array}{l}\operatorname{IgA-} \\
\text { lambda }\end{array}$ & 16 & $\begin{array}{l}\text { Parathyroidectomy, chemotherapy, and } \\
\text { radiotherapy }\end{array}$ & Hyperplasia & $\begin{array}{l}\text { Died } 1 \text { week } \\
\text { later }\end{array}$ & PHPT \\
\hline 29 & Our case & $92 \mathrm{~F}$ & IgG-kappa & 13.3 & $\begin{array}{l}\text { Bisphosphonates, Lasix, hydration, } \\
\text { calcitonin, melphalan, prednisone, } \\
\text { lenalidomide, and dexamethasone }\end{array}$ & - & Survived & Both \\
\hline
\end{tabular}

30 Johansson and Werner [29] mentioned 3 cases of MM and PHPT (no detail of the cases is given), one other such as has been described by Drezner and Lebovitz [30] without much detail.

* Calcium at time of diagnosis of MM.

patients had a combination of heavy and light chain MM, one patient had nonsecretory type of MM. All the patients had calcium $\geq 11 \mathrm{mg} / \mathrm{dL}$ at the time of presentation. Majority of patients had parathyroid adenoma as a cause of PHPT, few had chief cell hyperplasia, and none had parathyroid cancer. Parathyroidectomy, combination of radiotherapy, and chemotherapy had been used for treatment of this coexistent condition with variable success. Rao et al. [2] suggested that parathyroidectomy in patients with coexistent PHPT and MM serves three folds; first, it removes confusion about etiology of hypercalcemia; second, it alters prognosis of myeloma; third, calcium can be used as a tumor marker in cases if there is a recurrence of tumor. Considering age, our patient was not a candidate for surgery, in such patient population medical alternative to parathyroidectomy is needed. Ten out of 29-patients died within 5 years after codiagnosis, and out of those ten, eight died within one year.

\section{Conclusions}

A search for concomitant cause of hypercalcemia should be pursued in cases of difficult-to-control hypercalcemia and in elderly individuals, in whom the incidence of PTHP and MM is common.

\section{References}

[1] E. P. Pest, G. McQuaker, J. A. Hunter, D. Moffat, and A. J. Stanley, "Primary hyperparathyroidsm, amyloid and multiple myeloma: an unusual association," Scottish Medical Journal, vol. 50, no. 1, pp. 32-34, 2005.
[2] D. S. Rao, R. Antonelli, K. R. Kane, J. E. Kuhn, and C. Hetnal, "Primary hyperparathyroidism and monoclonal gammopathy," Henry Ford Hospital Medical Journal, vol. 39, no. 1, pp. 41-44, 1991.

[3] R. M. Jackson and M. J. Orland, "Parathyroid adenoma in a patient with multiple myeloma," Southern Medical Journal, vol. 72, no. 10, pp. 1336-1337, 1979.

[4] R. C. Chisholm, Y. J. Weaver, E. B. Chung, and J. L. Townsend, "Parathyroid adenoma and light chain myeloma," Journal of the National Medical Association, vol. 73, no. 9, pp. 875-880, 1981.

[5] R. M. Francis, A. G. Bynoe, and C. Gray, "Hypercalcaemia due to the coexistence of parathyroid adenoma and myelomatosis," Journal of Clinical Pathology, vol. 35, no. 7, pp. 732-736, 1982.

[6] R. J. Mundis and R. A. Kyle, "Primary hyperparathyroidism and monoclonal gammopathy of undetermined significance," American Journal of Clinical Pathology, vol. 77, no. 5, pp. 619$621,1982$.

[7] M. J. Stone, Z. H. Lieberman, Z. H. Chakmakjian, and J. L. Matthews, "Coexistent multiple myeloma and primary hyperparathyroidism," Journal of the American Medical Association, vol. 247, no. 6, pp. 823-824, 1982.

[8] D. R. Hoelzer and A. B. Silverberg, "Primary hyperparathyroidism complicated by multiple myeloma," Archives of Internal Medicine, vol. 144, no. 10, pp. 2069-2071, 1984.

[9] W. Schneider and M. Thomas, "Hypercalcaemia in coexistent parathyroid adenoma and multiple myeloma: problems of differential diagnosis," Deutsche Medizinische Wochenschrift, vol. 114, no. 31-32, pp. 1199-1202, 1989.

[10] E. Toussirot, F. Bille, J. F. Henry, and P. C. Acquaviva, "Coexisting kappa light chain multiple myeloma and primary hyperparathyroidism," Scandinavian Journal of Rheumatology, vol. 23, no. 1, pp. 49-50, 1994. 
[11] S. Goto, M. Yoshioka, K. Nagai et al., "Primary hyperparathyroidism associated with multiple myeloma," Internal Medicine, vol. 34, no. 10, pp. 988-991, 1995.

[12] F. Otsuka, N. Hayakawa, T. Ogura et al., "A case of primary hyperparathyroidism accompanying multiple myeloma," Endocrine Journal, vol. 44, no. 1, pp. 105-109, 1997.

[13] C. Fery-Blanco, C. Prati, P. Ornetti et al., "Hypercalcemia of double origin: association of multiple myeloma and ectopic parathyroidal adenoma," Revue de Medecine Interne, vol. 28, no. 7, pp. 504-506, 2007.

[14] E. Sarfati, P. de Ferron, C. Dubost, P. Assens, P. Veyssier, and B. Detour, "Multiple myeloma associated with primary hyperparathyroidism caused by an adenoma," Annales de medecine interne, vol. 136, no. 8, article 684, 1985.

[15] C. Rosen, H. Segal, C. E. Hartz, F. Mroz, and E. Carlton, "Primary hyperparathyroidism in an elderly patient with multiple myeloma," Journal of the American Geriatrics Society, vol. 40, no. 7, pp. 703-705, 1992.

[16] M. Tomon, M. Fukase, and M. Nakata, "A case of multiple myeloma associated with primary hyperparathyroidism," Hormone to Rinsho, vol. 37, article 128, 1989.

[17] Z. Fanari, H. Kadikoy, W. Haque, O. Pacha, and A. Abdellatif, "Medical management of primary hyperparathyroidism with concommitant multiple myeloma," Internal Medicine, vol. 49, no. 6, pp. 581-584, 2010.

[18] M. Bogas, L. Costa, and D. Araújo, "Coexistence of primary Hyperparathyroidism and multiple myeloma; association and rare manifestation," Acta Reumatologica Portuguesa, vol. 33, no. 1, pp. 98-105, 2008.

[19] Y. Katayama, H. Matsuda, Y. Katoh et al., "Multiple myeloma in a patient with primary hyperparathyroidism," Hinyokika Kiyo, vol. 35, no. 8, pp. 1369-1372, 1989.

[20] E. Romagnoli, S. Minisola, V. Carnevale, G. Spagna, E. D’Erasmo, and G. Mazzuoli, "Coexistent multiple myeloma and MEN type 1," Postgraduate Medical Journal, vol. 66, no. 780, pp. 879-880, 1990.

[21] V. Toh and P. Winocour, "Multiple myeloma with hyperparathyroidism," Hospital Medicine, vol. 61, no. 10, article 744, 2000.

[22] B. Sopeña, G. J. Rodríguez, J. De La Fuente, and C. MartínezVázquez, "Two causes of hypercalcemia: learning by the holmesian method," Mayo Clinic Proceedings, vol. 79, no. 5, article 708, 2004.

[23] H. M. Khandwala and M. A. Boctor, "Multiple myeloma presenting with recurrent hypercalcemia in a patient with a history of primary hyperparathyroidism: report of case and review of literature," Endocrine Practice, vol. 10, no. 4, pp. 345347, 2004.

[24] N. Patel, A. Talwar, L. Donahue, V. John, and D. Margouleff, "Hyperparathyroidism accompanying multiple myeloma," Clinical Nuclear Medicine, vol. 30, no. 8, pp. 540-542, 2005.

[25] B. Avcioglu, T. Bayraktaroglu, A. Kubat Uzum et al., "A case with hypercalcemia caused by hyperparathyroidism and multiple myeloma," Endocrine Abstracts, vol. 14, article 458, 2007.

[26] F. U. Chowdhury and A. F. Scarsbrook, "Tc-99m sestamibi uptake mimicking parathyroid adenoma in a patient with primary hyperparathyroidism and occult multiple myeloma," Clinical Nuclear Medicine, vol. 33, no. 3, pp. 198-200, 2008.

[27] A. G. Dalgleish and P. A. Gatenby, "Refractory hypercalcaemia: parathyroid adenoma or multiple myeloma?" Medical Journal of Australia, vol. 140, no. 2, pp. 99-100, 1984.
[28] K. M. Peters, J. Rosenberger, A. Gaczkowski, and R. Lorenz, "Concomitant occurrence of hyperparathyroidism and multiple myeloma," Internist, vol. 30, no. 2, pp. 117-119, 1989.

[29] H. Johansson and I. Werner, "Dysproteinemia, malignancy, and hyperparathyroidism," Annals of Internal Medicine, vol. 83, no. 1, pp. 121-122, 1975.

[30] M. K. Drezner and H. E. Lebovitz, "Primary hyperparathyroidism in paraneoplastic hypercalcaemia," The Lancet, vol. 1, no. 8072, pp. 1004-1006, 1978.

[31] S. French, J. Subauste, and S. Geraci, "Calcium abnormalities in hospitalized patients," Southern Medical Journal, vol. 105, pp. 231-237, 2012.

[32] R. A. Kyle, M. A. Gertz, T. E. Witzig et al., "Review of 1027 patients with newly diagnosed multiple myeloma," Mayo Clinic Proceedings, vol. 78, no. 1, pp. 21-33, 2003.

[33] G. R. Mundy, T. Yoneda, and T. A. Guise, "Hypercalcemia in hematologic malignancies and in solid tumors associated with extensive localized bone destruction," in Primer on the Metabolic Bone Diseases and Disorders of Mineral Metabolism, M. J. Favus, Ed., pp. 208-211, Lippincott Williams \& Wilkins, Philadelphia, Pa, USA, 4th edition, 1999.

[34] H. Tsujimura, F. Nagamura, T. Iseki, S. Kanazawa, and H. Saisho, "Significance of parathyroid hormone-related protein as a factor stimulating bone resorption and causing Hypercalcemia in myeloma," American Journal of Hematology, vol. 59, pp. 168-170, 1998.

[35] R. Kitazawa, S. Kitazawa, K. Kajimoto et al., "Expression of parathyroid hormone-related protein (PTHrP) in multiple myeloma," Pathology International, vol. 52, no. 1, pp. 63-68, 2002.

[36] R. John, D. Oleesky, B. Issa et al., "Pseudohypercalcaemia in two patients with IgM paraproteinaemia," Annals of Clinical Biochemistry, vol. 34, no. 6, pp. 694-696, 1997.

[37] J. M. Van Dijk, M. Sonnenblick, N. Weissberg, and A. Rosin, "Pseudohypercalcemia and hyperviscosity with neurological manifestations in multiple myeloma," Israel Journal of Medical Sciences, vol. 22, no. 2, pp. 143-144, 1986.

[38] J. S. Clubb, S. Posen, and F. C. Neale, "Disappearance of a serum paraprotein after Parathyroidectomy," Archives of internal medicine, vol. 114, pp. 616-620, 1964.

[39] A. Bellou, H. Blain, A. Guerci, and C. Jeandel, "Gammapathie monoclonale et hyperparathyroïdie primitive. À propos de deux observations et revue de la littérature," Revue de Medecine Interne, vol. 17, no. 4, pp. 325-328, 1996.

[40] B. Arnulf, D. Bengoufa, E. Sarfati et al., "Prevalence of monoclonal gammopathy in patients with primary hyperparathyroidism: a prospective study," Archives of Internal Medicine, vol. 162, no. 4, pp. 464-467, 2002.

[41] F. Q. Pirih, M. N. Michalski, S. W. Cho et al., "Parathyroid hormone mediates hematopoietic cell expansion through interleukin-6," PLoS One, vol. 5, article 13657, 2010.

[42] E. M. Greenfield, S. M. Shaw, S. A. Gornik, and M. A. Banks, "Adenyl cyclase and interleukin 6 are downstream effectors of parathyroid hormone resulting in stimulation of bone resorption," Journal of Clinical Investigation, vol. 96, no. 3, pp. 12381244, 1995.

[43] J. R. Luckasen, J. G. White, and J. H. Kersey, "Mitogenic properties of a calcium ionophore, A23187," Proceedings of the National Academy of Sciences of the United States of America, vol. 71, no. 12, pp. 5088-5090, 1974. 
[44] E. B. Lewis, "Leukemia, multiple myeloma, and aplastic anemia in american radiologists," Science, vol. 142, no. 3598, pp. 14921494, 1963.

[45] A. B. Schneider, T. C. Gierlowski, E. Shore-Freedman, M. Stovall, E. Ron, and J. Lubin, "Dose-response relationships for radiation-induced hyperparathyroidism," Journal of Clinical Endocrinology and Metabolism, vol. 80, no. 1, pp. 254-257, 1995. 


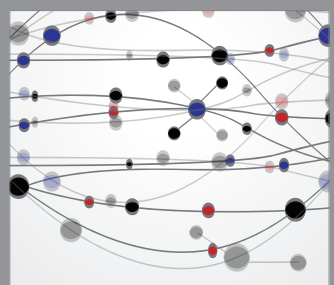

The Scientific World Journal
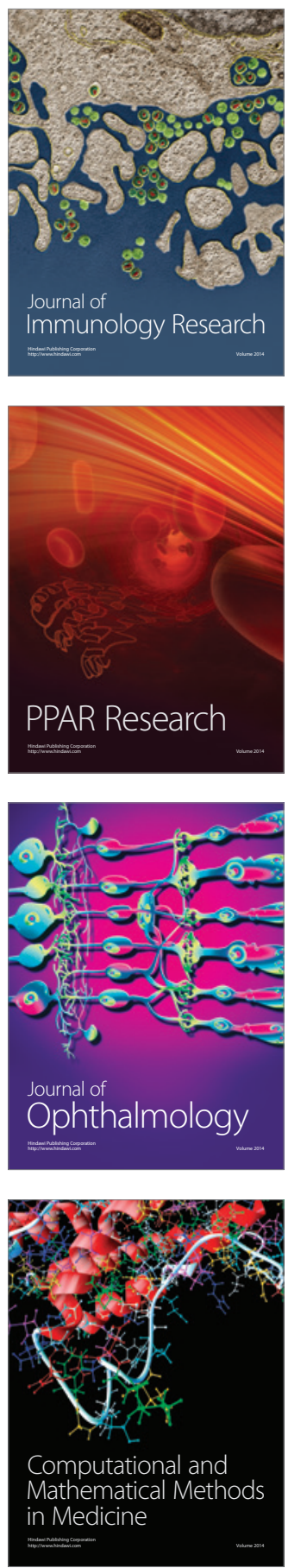

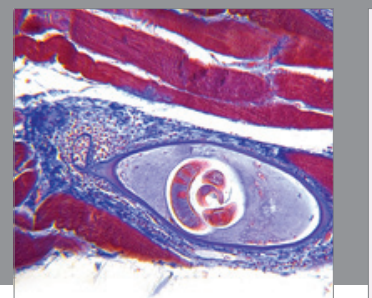

Gastroenterology

Research and Practice
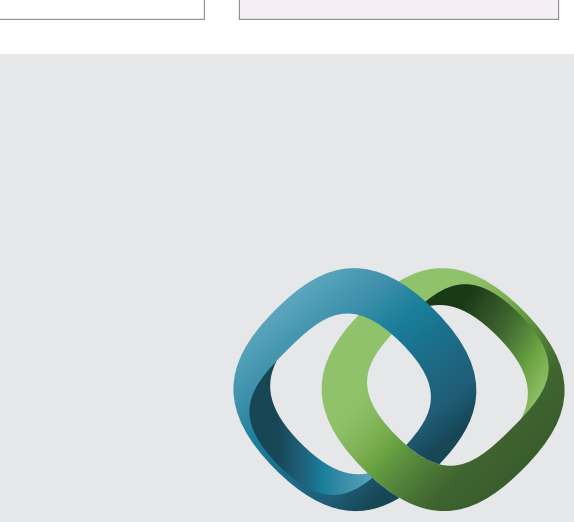

\section{Hindawi}

Submit your manuscripts at

http://www.hindawi.com
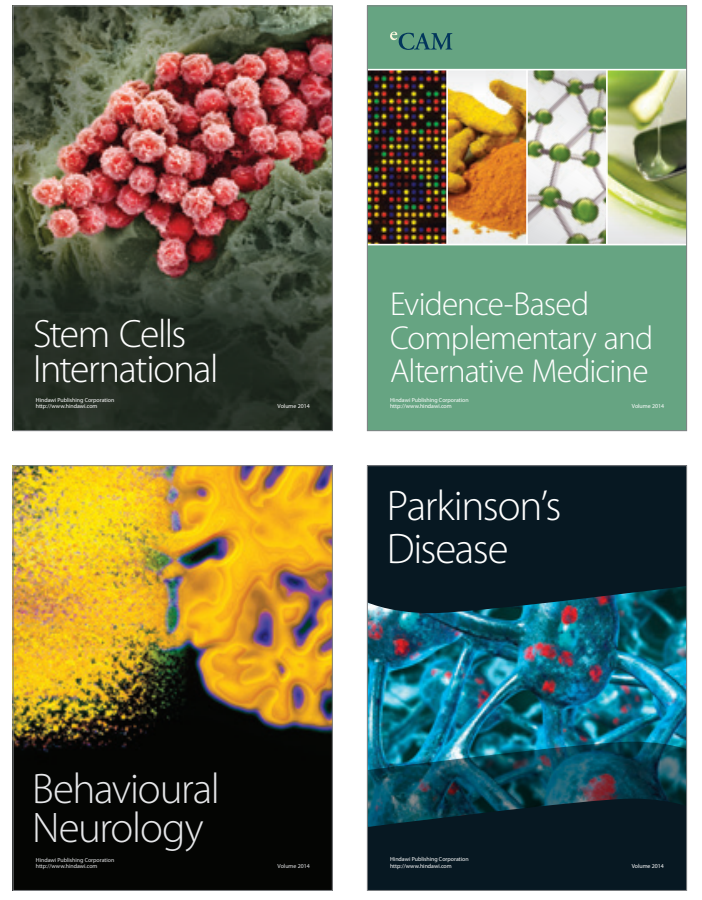
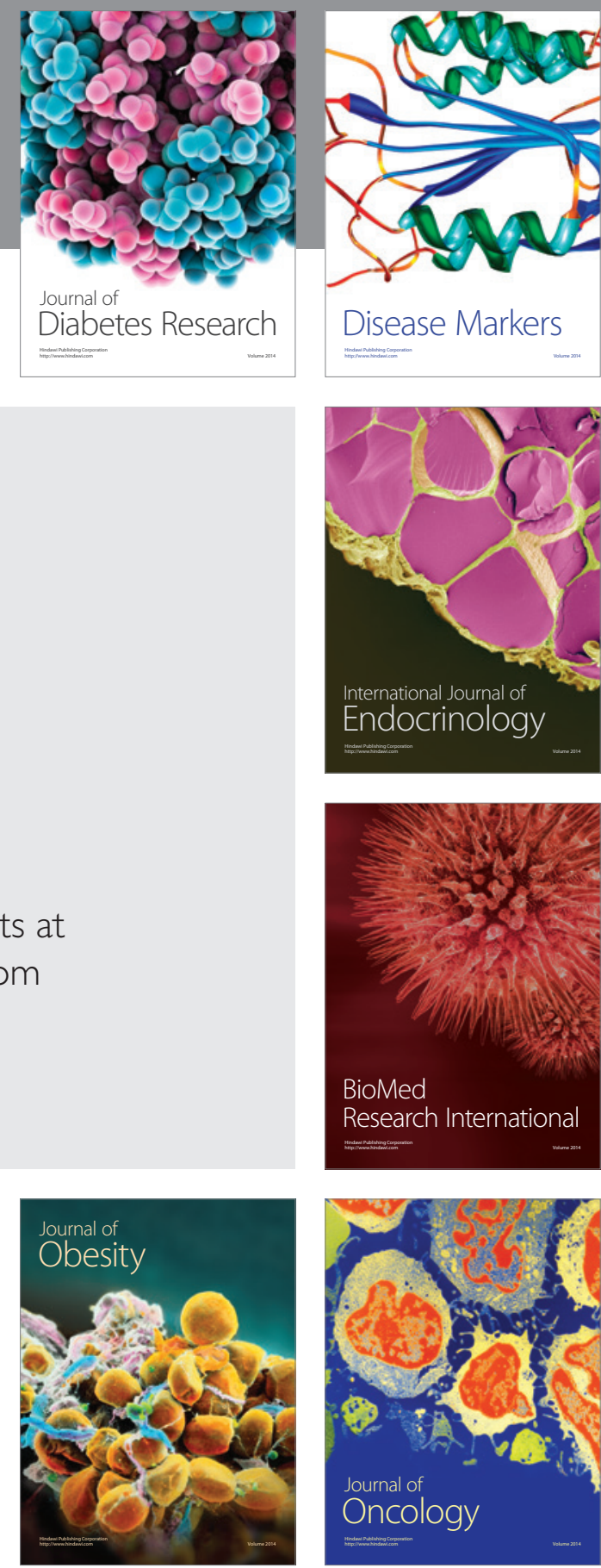

Disease Markers
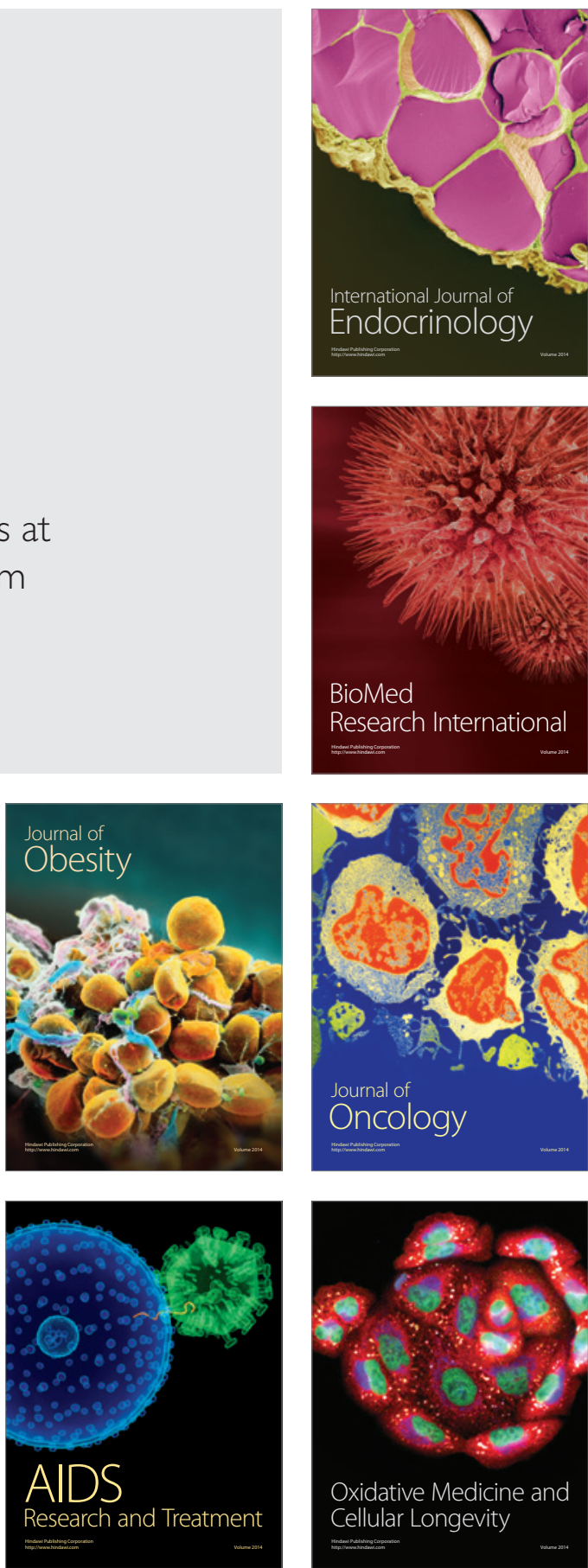